\title{
A point prevalence surveillance study from pediatric and neonatal specialty hospitals in India
}

\author{
Sanjeev Singh ${ }^{\mathrm{a}, *}$, Tency Jose ${ }^{\mathrm{a}}$, Ann Versporten $^{\mathrm{b}}$, Sharmila Sengupta ${ }^{\mathrm{c}}$, P. Fini $^{\mathrm{a}}$, Mike Sharland ${ }^{\mathrm{d}}$, \\ R. Krishna Kumar a and Herman Goossens ${ }^{\mathrm{b}}$ \\ ${ }^{a}$ Amrita Institute of Medical Sciences, Kochi, Kerala, India \\ ${ }^{\mathrm{b}}$ Department of Clinical Microbiology, University of Antwerp, Antwerp, Belgium \\ ${ }^{\mathrm{c}}$ BL Kapoor Hospital, New Delhi, India \\ dPaediatric Infectious Diseases Research Group, St George's University of London, London, UK
}

Received 24 August 2014

Revised 8 October 2014

Accepted 14 October 2014

\begin{abstract}
Point prevalence surveys (PPS) have been used to document antimicrobial use in healthcare organizations. Antimicrobial resistance is a global concern, more so for developing countries like India, where the burden of antimicrobial resistance and infectious diseases are very high. A web based PPS developed by University of Antwerp, Belgium for the Antibiotic Resistance and Prescribing in European Children (ARPEC) project was used to enter data from pediatric and neonatal wards and intensive care units (ICU) from 8 centers across India. All patients who were receiving systemic antibacterial treatments on the day of the survey inclusive of antibacterial prophylaxis for surgery were included. 195 patients were surveyed across India: 105 (54\%) medical and $90(46 \%)$ surgical. 192 (98\%) patients received multiple antimicrobials out of which 187 (95\%) were given parenterally. Empirical antimicrobials were prescribed in $138(71 \%)$ patients and targeted therapy in 57 (29\%). The commonest infections for which antimicrobials were prescribed were healthcare associated infections 180 (41.76\%) followed by surgical prophylaxis $90(21 \%)$ and community acquired infections $84(18 \%)$. Antimicrobials usage was maximal in ICUs. More than a single dose $(5.6 \%)$ for more than a day $(86.7 \%)$ was used for surgical prophylaxis. The Web based PPS suggests widespread antibiotic usage among pediatric and neonatal patients in selected tertiary Indian hospitals and underscores the need for antibiotic stewardship in order to promote rational and evidence based practice to limit the emergence of antibiotic resistant microbes.
\end{abstract}

Keywords: Point prevalence surveys, antimicrobial resistance, prophylaxis

\section{Introduction}

Healthcare associated infections (HAI) are a recognized public health problem worldwide and contribute significantly to the morbidity, mortality and cost of care. HAI places a burden to the limited healthcare resources in developing countries and puts

*Correspondence: Dr. Sanjeev Singh, A 12, EKTA, Amrita Institute of Medical Sciences, Ponekara, Kochi, 682041, India. Tel.: +91 484 2801234; Fax: +91 484 2802121; E-mail: sanjeevksingh@ aims.amrita.edu. patient's safety at risk [1]. There are multiple factors that contribute towards the risk of HAI. They include immuno-compromised host, neonatal and geriatric population, and prolonged hospital stay, use of complex procedures, indwelling lines, feeding tubes and emerging antimicrobial resistance [2].

Antimicrobial resistance is a huge concern in present day practice. The treatment options are limited with resultant increase in morbidity, mortality and costs [3]. The unwarranted and unrestricted use of antimicrobial treatment is responsible for widespread 
antimicrobial resistance.

Antibiotic stewardship is the foundation for strong and robust processes to improve antimicrobial prescribing and prevent antimicrobial resistance. Good antimicrobial stewardship involves selecting appropriate drugs and optimizing doses and duration of therapy for successful treatment, while minimizing toxicity and selection of resistant bacterial strains.

Point prevalence surveys (PPS) are a useful tool to measure and monitor the use of antimicrobials, burden of HAI and antimicrobial resistance. PPS is a mechanism through which cross sectional information across participating hospitals can be captured providing a snapshot of practice pattern. PPS as a tool can be used to understand the burden of all types of HAIs, trends and efficacy of infection control measures [4]. PPS can provide a sound contextually appropriate rationale for antibiotic stewardship programs (ASP).

\section{Methods}

The European project "Antibiotic Resistance and Prescribing in European Children (ARPEC)" point prevalence surveillance was initiated among 191 centers across the globe. The laboratory of Medical Microbiology (UA, Belgium) designed the ARPEC web PPS (http://app.esac.ua.ac.be/arpec_webpps/). The ARPEC work package focused on the collection of consistent data on antimicrobials (antibacterials, antivirals and antifungals) administered to pediatric and neonatal patients.

Pre-designed ARPEC Pediatrics and Neonatal PPS Survey forms were used. Essential data were the patients age, gender, weight, antimicrobial agent, dose per administration, number of doses per day, route of administration, antimicrobial agent, underlying diagnosis, site of infection in accordance to a list of provided, reasons for treatment and indication for therapy (community acquired, versus hospital acquired infection or prophylaxis).

In non-European regions hospitals were asked to plan PPS in November 2012. A month was given to enter the data using the web-based data entry program. One day was identified for conducting PPS on all existing pediatric and neonatal intensive care units (ICU), high dependency units (HDU) and wards. Every ward (pediatric and neonatal) was involved during this survey. Each hospital conducted a pilot first to select the most appropriate day for data collec-

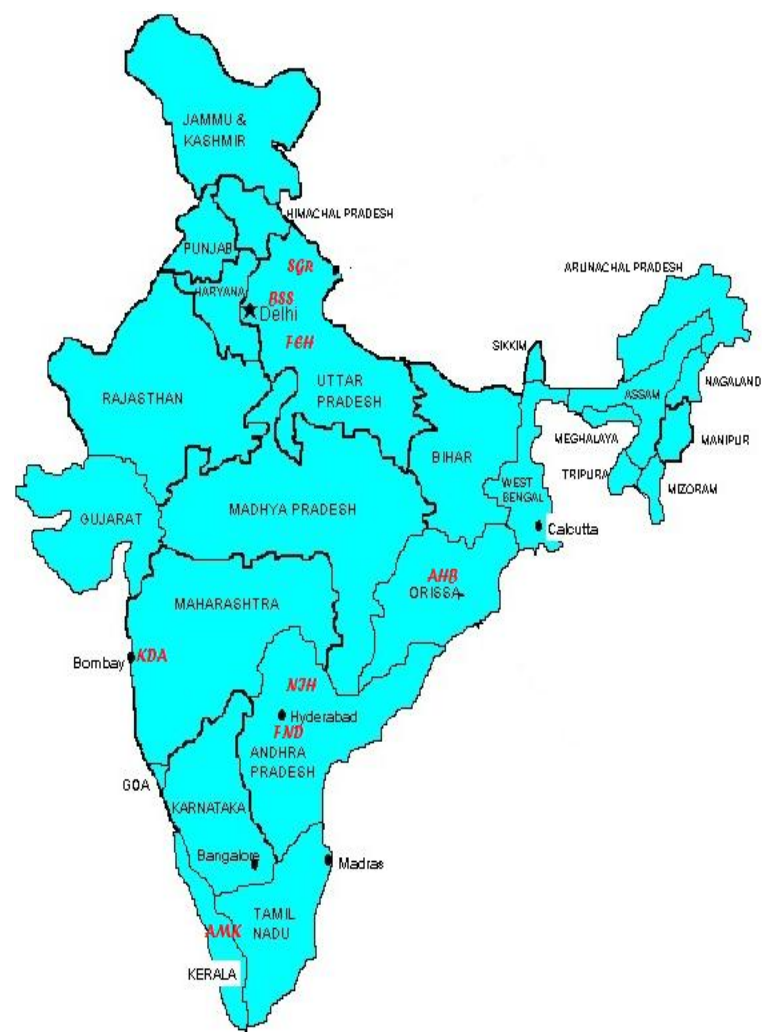

Fig. 1. Overall representation of participating center across the globe. (Colours are visible in the online version of the article; http://dx.doi.org/ 10.3233/JPI-140429)

tion and entry [5].

In India 8 private hospitals were selected (Fig. 1). All of them were running level 3 NICU. Out of 8 , one was a university teaching hospital, 2 were exclusive paediatric hospitals and rest were multispecialty healthcare organizations (HCOs). All HCOs were admitting premature, very low birth weight babies from their busy high risk obstetrics facility. All HCOs had a dedicated paediatric surgical service which dealt with complex paediatric surgical admissions.

All patients less than 18 years admitted in pediatric wards and all neonatal patients admitted to NICU at 8 am on the morning of the survey who received an antimicrobial during the 24 hours prior to the survey were included. Patients admitted to or transferred into the ward after 8 am were excluded. Patients who left the ward before the survey were excluded from the survey. Emergency surgical admissions and out-patients were excluded [6].

The reasons for antimicrobial prescription (empirical, targeted or prophylaxis) and the underlying diagnoses were collected. 
Table 1

Patient Characteristics at each location and treatment modality

\begin{tabular}{lcc}
\hline & Pediatric N (\%) & Neonatal (\%) \\
\hline Male & $83(61.4)$ & $41(68.3)$ \\
Female & $52(38.5)$ & $19(31.6)$ \\
ICU & $56(41.8)$ & $54(90)$ \\
Ward & $79(58.5)$ & $6(10)$ \\
Hospital acquired infection & $90(66.66)$ & $51(85)$ \\
Community acquired infection & $45(33.33)$ & $9(15)$ \\
Medical prophylaxis & $92(68.1)$ & $57(95)$ \\
Surgical prophylaxis & $43(31.85)$ & $3(5)$ \\
Treatment & $33(24.4)$ & $13(21.6)$ \\
Prophylaxis & $102(75.5)$ & $47(78.3)$ \\
\hline
\end{tabular}

Data were anonymized and entered into the web PPS.

ID fellows, microbiologists, pharmacists, infection control nurses and an administrator were involved in the study. HAI definition was derived from the European Center for Disease Control (ECDC) and agreed upon by all participating centers. Descriptive analysis was carried out by using SPSS Version 17 and Stata Version 9.

\section{Results}

A total of 195 patients from 8 hospitals across India were included in the survey (Fig. 1). 105 (54\%) medical patients and $90(46 \%)$ surgical patients were included from a number of different wards. 60 (30.7\%) patients were neonates and 135 (69.2\%) pediatric. Out of 135 paediatric patients, 65 (48.1\%) were admitted in Paediatric Intensive Care (PICU). $64.43 \%(n=122)$ were males (Table 1$)$.

Out of 195 patients, 192 (98.4\%) were prescribed multiple antimicrobials (e.g., amikacin, piperacillin tazobactam and fluconazole). 187 patients were administered parenteral antimicrobials and only $8(4 \%)$ patients were on oral antibiotics Out of 105 medical patients, there were 138 (71\%) prescriptions for empirical therapy and $57(29.2 \%)$ for targeted therapy (Table 1). Out of 431 doses, $180(41.76 \%)$ were prescribed for HAI, 90 (20.9\%) for surgical prophylaxis, $342(79.3 \%)$ for medical prophylaxis $84(17.86 \%)$ for community acquired infections and $77(16.5 \%)$ for medical prophylaxis. The top ten most commonly prescribed antimicrobials accounted for $60 \%$ of all antimicrobials prescribed for treatment of infection ( $n$ $=295$ ). The top three antimicrobials prescribed were amikacin 58 (13.5\%), piperacillin-tazobactam 45 (10.4\%), fluconazole $36(8.4 \%)$ (Table 2$)$.
Table 2

Commonest antibiotics prescribed for different indications

\begin{tabular}{lll}
\hline Community acquired & Piperacillintazobactam & $13(3.01 \%)$ \\
infection & Ceftriaxone & $12(2.78 \%)$ \\
Hospital acquired & Amikacin & $10(2.3 \%)$ \\
infection & Meropenem & $27(6.2 \%)$ \\
& Piperacillintazobactam & $20(4.6 \%)$ \\
Oral & Fluconazole & $13(3.0 \%)$ \\
& Fluconazole & $12(2.7 \%)$ \\
Parental & Co-amoxiclavulanic acid & $4(0.92)$ \\
& Amikacin & $58(13.45)$ \\
Empirical & Meropenem & $34(7.88 \%)$ \\
& Piperacillintazobactam & $45(10.4)$ \\
Targeted & Amikacin & $52(12.06 \%)$ \\
& Piperacillintazobactam & $36(8.35 \%)$ \\
& Fluconazole & $27(6.26 \%)$ \\
Medical prophylaxis & Meropenem & $19(4.4 \%)$ \\
& Amikacin & $6(1.3 \%)$ \\
& Cefotaxime & $6(1.3 \%)$ \\
Surgical prophylaxis & Amikacin & $41(11.8 \%)$ \\
& Fluconazole & $36(10.5 \%)$ \\
& Amikacin & $35(10.2 \%)$ \\
& Cefuroxime & $22(24.5 \%)$ \\
& Cefotaxime & $20(22.47 \%)$ \\
\hline
\end{tabular}

Table 3

Comparing prescription patterns in India and Europe - pediatric and neonatal pateints

\begin{tabular}{lcc}
\hline & India $(n ; \%)$ & Europe $(n ; \%)$ \\
\hline Paediatric patients & & \\
318 prescriptions, 135 & patients & \\
Intravenous Therapy & $213(67 \%)$ & $2115(72 \%)$ \\
Oral Drug & $38(12 \%)$ & NA \\
Multiple antibiotics & $67(21 \%)$ & $976(33 \%)$ \\
Neonatal patients & & \\
113 prescriptions, 60 patients & \\
Multiple antibiotics & $31(27.5 \%)$ & $470(70 \%)$ \\
\hline
\end{tabular}

The prevalence of HAI was highest in HDU/ICU. The most commonly occurring HAI was sepsis $(n=$ 74) followed by lower tract respiratory infection (LRTI) $(n=31)$ urinary tract infections $(n=9)$ and gastrointestinal tract infection $(n=7)$. These four groups accounted for nearly $60 \%$ of all HAIs.

For surgical prophylaxis, the three most commonly prescribed antimicrobials were amikacin $24.7 \%(n=$ $22)$ followed by cefuroxime $23.5 \%(n=21)$ and cefotaxime $14.6 \%(n=13)$. This accounted for $70 \%$ of all antimicrobial prescribed for surgical prophylaxis. 5 patients $(5.61 \%)$ were given single doses as per the institutional guidelines, 7 patients $(7.86 \%)$ received surgical prophylaxis for $24 \mathrm{hrs}$ and 78 patients $(89 \%)$ received antibiotics for more than a day (Table 3 ). 
The number of patients who were receiving antimicrobials for HAI were compared with similar setting in Europe, meeting the same epidemiological case definitions for HAI (Table 3) [5-7].

\section{Discussion}

Antimicrobial resistance is a stark reality across the globe, more so in India [8]. PPS is a tool for capturing information on antimicrobial prescription practices [10]. We report the first PPS conducted among paediatric and neonatal patients across 8 centers in India. The maximum use of antimicrobials was seen in ICU/HDU settings for healthcare associated infections. This suggests the immediate need for adoption of good infection control practices and evidence based antimicrobial therapy. The most common indication for antimicrobial therapy was HAI, followed by surgical prophylaxis, community acquired infections and medical prophylaxis. Almost all patients received multiple antibiotics through a parental route. $70 \%$ of the patients were prescribed multiple antibiotics on empirical basis, indicating the need for appropriate guidelines.

There was high use of antimicrobials for medical prophylaxis: amikacin, piperacillin-tazobactam and flucanazole were the top 3 antimicrobials prescribed. Antimicrobials selected for surgical prophylaxis were prescribed for more than the single recommended dose and sometimes for more than 24 hours. There was a high percentage of community acquired infections admitted in ICUs/HDUs possibly because all centers selected for PPS were tertiary care referral centers receiving complex patients from peripheral institutions.

There were striking differences in antimicrobial prescription patterns between Indian and European centers. European centers used narrow spectrum antimicrobials consisting mainly of penicillins, sulphonamides, aminoglycosides and other betalactams, while in the Indian centers high-end reserve antibiotics like piperacillin-tazobactam and carbapenems were commonly used [7-9].

Globally antimicrobial surveillance is the backbone of successful programs to reduce antimicrobials resistance and address overuse and misuse of antimicrobials [11]. Adequate surveillance of antimicrobials resistance and antibacterial use in resource limited settings needs to be implemented more widely. Im- mediate priority should be given to develop antibiotic stewardship program (ASP) in India. ASP will help capacity building by continually educating healthcare professionals about rational and evidence based antimicrobials use [11]. Adequate understanding of the local resistance patterns and emerging resistance is essential.

PPS collect data of a single day and could have several potential limitations as may not truly reflect the reality. Repeat PPS collection days across the participating centers and more frequent collections would yield better representation. Notwithstanding this limitation, it is quite apparent that the antimicrobials usage in India makes greater use of high-end antibiotics and multiple antimicrobials. The duration of surgical prophylaxis is inappropriately long in most instances. The results of this survey indicates that there is an urgent need to introduce a systematic antibiotic stewardship program nationally together with development of stringent guidelines and policies to limit antimicrobials overuse guided by a good knowledge of the patterns of antimicrobial resistance.

\section{Acknowledgements}

Dr. Praveen Kumar of PGIMER, Chandigarh, Dr. Neelam Kler of Gangaram Hospital, New Delhi, Dr. Sasidharan of Amrita Institute of Medical Sciences, Kochi, Dr. Bhavani K of NICE hospital, Hyderbad, Dr. Srinivas Murki of Fernandez Hospital, Hyderabad, Dr. Sharmila Sengupta of BL Kapoor Hospital, New Delhi, Dr. Tanu Singhal of Kokilaben Ambani Hospital, Mumbai, Dr. Sunita Sahu of Apollo Hospital, Bhusneshwar, Dr. Abha Mehndiratta, Access Healthcare International, New Delhi.

\section{Conflicts of interest}

None.

\section{Funding}

Nil.

\section{References}

[1] World Health Organisation. Report on the Burden of Endemic 
Healthcare Associated Infections worldwide. Geneva: WHO; 2011.

[2] European Center for Disease Prevention and Control. Antimicrobial resistance surveillance in Europe 2010. Annual report of the European Antimicrobial Resistance Surveillance Network. Stockholm: ECDC 2011.

[3] Mölstad S, Erntell M, Hanberger H, Melander E, Norman C, Skoog G, Lundborg CS, Söderström A, Torell E, Cars O. Sustained reduction of antibiotic use and low bacterial resistance: 10-year follow-up of the Swedish Strama programme. Lancet Infect Dis 2008; 8(2): 125-32.

[4] French GL, Cheng AF, Wong SL, Donnan S. Repeated prevalence surveys for monitoring effectiveness of hospital infection control. Lancet 1989; 2(8670): 1021-3.

[5] Health Protection Scotland. Scottish National Prevalence Survey of Healthcare Associated Infection and Antimicrobial Prescribing Data Collection Protocol v1. Glasgow: Health Protection Scotland; 2011.

[6] Information Service Division Scotland [homepage on the Internet]. Name of the city: Hospital Classification. Information Services Division 2011 [cited 2012 Mar 22]; Available from: http://www.isdscotland.org/isd/servlet/FileBuffer?namedFile $=$ Costs_Hospital_"ttp://www.isdscotland.org/isd/servlet/File Buffer?namedFile=Costs_Hospital_Class.xls\&pContentDisp ositionType $=$ inline
[7] Versporten A, Sharland M, Bielicki J, Drapier N, Vankerckhoven V, Goossens H; ARPEC Project Group Members. The antibiotic resistance and prescribing in European Children project: a neonatal and pediatric antimicrobial web-based point prevalence survey in 73 hospitals worldwide. Pediatr Infect Dis J 2013; 32(6): e242-53.

[8] Ghafur A, Mathai D, Muruganathan A, Jayalal JA, Kant R, Chaudhary D et al. The Chennai Declaration: a roadmap to tackle the challenge of antimicrobial resistance. Indian $\mathbf{J}$ Cancer 2013; 50(1): 71-3.

[9] Reilly JS, Price L, Godwin J, cairns S, Hopkins S, Cookson B et al. Pilot validation study of the ECDC point prevalence survey of healthcare associated infections and antmicrobial use in European acute care hospitals, Glasgow: Glasgow Caledonian University; 2012.

[10] Malcolm W, Cromwell T. Scottish Antimicrobial Prescribing Group European Surveillance of Antimicrobial Consumption Point Prevalance Survey 2009 Scottish Hospitals Report. Scotland: Health Protection Scotland; Information Services Division; 2012.

[11] Public Health update of Carbapenum-Resistant Enterobacteriaceae (CRE) producing metallo-beta-lactamases in the US reported to CDC. Center for Disease Control and Prevention 2011 (cited 2012 Mar 27). 